\title{
Molecular Dynamics Study of Contact Mechanics: Contact Area and Interfacial Separation from Small to Full Contact
}

\author{
C. Yang and B. N. J. Persson \\ Institut für Festkörperforschung, Forschungszentrum Jülich, D-52425 Jülich, Germany
}

(Received 27 September 2007; published 16 January 2008)

\begin{abstract}
We report a molecular dynamics study of the contact between a rigid solid with a randomly rough surface and an elastic block with a flat surface. We study the contact area and the interfacial separation from small contact (low load) to full contact (high load). For small load the contact area varies linearly with the load and the interfacial separation depends logarithmically on the load. For high load the contact area approaches the nominal contact area (i.e., complete contact), and the interfacial separation approaches zero. The present results may be very important for soft solids, e.g., rubber, or for very smooth surfaces, where complete contact can be reached at moderate high loads without plastic deformation of the solids.
\end{abstract}

DOI: 10.1103/PhysRevLett.100.024303

It is very difficult to prepare surfaces which are really flat. Even on the most polished surfaces, hills and valleys are present which are large compared with the atomic-size. Usually, if two solids are placed in contact, the upper surface will be supported on the summits of the irregularities, and large surface areas will be separated by distances which are great compared with the molecular range of action [1-4]. The separation $u(\mathbf{x})$ between the surfaces will vary in a nearly random way with the lateral coordinates $\mathbf{x}=(x, y)$ in the apparent contact area. When the applied squeezing pressure increases, the contact area $A$ will increase and the average surface separation $\bar{u}=\langle u(\mathbf{x})\rangle$ will decrease, but in most situations it is not possible to squeeze the solids into perfect contact corresponding to $\bar{u}=0$. Understanding the area of real contact, and the interfacial separation between two solids is essential to friction, adhesion, sealing and many other important applications [5-8].

Most studies of contact mechanics have been focused on a small load where the contact area depends linearly on the load [9-15]. However, for soft solids, such as rubber or gelatin, or for smooth surfaces, nearly full contact may occur at the interface; therefore, it is of great interest to study how the contact area, the interfacial surface separation and stress distribution vary with load from small load (where the contact area varies linearly with the load from a small load), to high load [where the contact is (nearly) complete]. Here we will present such a study using molecular dynamics (MD), and we will compare the numerical results with the prediction of the analytical contact mechanics theory of Persson [16-18].

We consider the frictionless contact between elastic solids with randomly rough surfaces. If $z=h_{1}(\mathbf{x})$ and $h_{2}(\mathbf{x})$ describe the surface profiles, $E_{1}$ and $E_{2}$ are the Young's elastic moduli of the two solids and $\nu_{1}$ and $\nu_{2}$ the corresponding Poisson ratios, then the elastic contact problem is equivalent to the contact between a rigid solid (substrate) with the roughness profile $h(\mathbf{x})=h_{1}(\mathbf{x})+$
PACS numbers: 46.55.+d, 46.05.+b, 62.20.Qp

$h_{2}(\mathbf{x})$, in contact with an elastic solid (block) with a flat surface and with an Young's modulus $E$ and Poisson ratio $\nu$ chosen so that [19]

$$
\frac{1-\nu^{2}}{E}=\frac{1-\nu_{1}^{2}}{E_{1}}+\frac{1-\nu_{2}^{2}}{E_{2}} .
$$

Here we define $E^{*}=E /\left(1-\nu^{2}\right)$ as effective elastic modulus.

Consider randomly rough surfaces with roughness wavelength components in some finite range $\lambda_{1}<\lambda<$ $\lambda_{0}$, where $\lambda_{0}$ is comparable to (but smaller than) the lateral size of the nominal contact area. In order to accurately describe the contact mechanics between elastic blocks, it is necessary to consider a solid block which extends (at least) a distance $\sim \lambda_{0}$ in the direction normal to the nominal contact area. This leads to an enormous number of atoms or dynamical variables even for a small systems. In order to avoid this trouble we have developed a multiscale MD approach [12]. The atoms at the interface between the block and substrate interact with the repulsive potential $U(r)=\epsilon\left(r_{0} / r\right)^{12}$, where $r$ is the distance between a pair of atoms, $r_{0}=3.28 \AA$ and $\epsilon=74.4 \mathrm{meV}$. In the MD-model calculations there is no unique way to define the separation $\bar{u}$ between the solid walls. Here we have used the same definition as in Ref. [12] $\bar{u}=d-d_{c}$, where $d$ is the difference between the plane through the center of the atoms of the top layer of substrate atoms and bottom layer of block atoms. $d_{c}$ is the critical atom-atom separation used to define contact on the atomic scale. Thus, $\bar{u}=0$ corresponds to the separation $d_{c}=4.36 \AA$ between planes through the center of the interfacial atoms of the block and the substrate.

The system has lateral dimension $L=N a$, where $a$ is the lattice space of the block. Periodic boundary condition is used in $x y$ plane. For the block $N=400$, while the lattice space of the substrate $b \approx a / \phi$, where $\phi=(1+$ $\sqrt{5}) / 2$ is the golden mean, in order to avoid the formation of commensurate structures at the interface. The mass of 
the block atoms is 197 a.m.u. and $a=2.6 \AA$, reproducing the atomic mass and density of gold. The elastic modulus and Poisson ratio of the block is $E=77.2 \mathrm{GPa}$ and $\nu=$ 0.42 , respectively. The substrate surface has self-affine fractal surface roughness $[12,20]$. For a self-affine fractal surface the power spectrum has power-law behavior $C(q) \sim q^{-2(H+1)}$, where the Hurst exponent $H$ is related to fractal dimension $D_{f}$ of the surface via $H=3-D_{f}$. For real surfaces this relation holds only for a finite wave vector region $q_{0}<q<q_{1}$. Note that in many cases there is roll-off wave vector $q_{0}$ below which $C(q)$ is approximately constant. The randomly rough substrates we use have been generated as described in Ref. [20], and have root-meansquare roughness $h_{\text {rms }}=10 \AA$, fractal dimension is $D_{f}=$ 2.2, and roll-off wave vector $q_{0}=3 q_{L}$, where $q_{L}=2 \pi / L$, $L=1040 \AA$. We define the magnification $\zeta=q_{1} / q_{0}$. The contact between block and substrate, has been prepared in this way: First, the two surfaces are separated in a large distance, so that the block atoms feel no interaction, or weak interaction from substrate atoms. Second, the block is brought down slowly to substrate in a stepwise way (i.e., at different positions), so that the block can feel stronger interaction from substrate when it approaches to substrate. Then one can bring those configurations at different positions into equilibrium. Finally, one can get the corresponding pressure, contact area and interfacial separation at each equilibrated state.

With molecular dynamics simulations we can calculate the interfacial stress distribution. In order to obtain the contact area we follow the procedure outlined in Ref. [12] and fit the numerical results to the theoretically predicted stress distribution

$$
P(\sigma, \zeta)=\frac{1}{2(\pi \tilde{G})^{1 / 2}}\left(e^{-(\sigma-p)^{2} / 4 \tilde{G}}-e^{-(\sigma+p)^{2} / 4 \tilde{G}}\right),
$$

where $\tilde{G}(p, \zeta)$ depends on the nominal squeezing pressure $p$ and the magnification $\zeta$ (but which is independent of the interfacial stress $\sigma$, see below), and which we choose to get the best fit with the numerical data. In Fig. 1 we have shown the good agreement between the numerical pressure distribution and the analytical theory (for $\zeta=4$ ) under three different nominal pressures. Once $\tilde{G}$ is known we can calculate the relative contact area using [21]

$$
\frac{A}{A_{0}}=\int_{0}^{\infty} d \sigma P(\sigma, \zeta)
$$

In Fig. 2(b) we show the relative contact area $A / A_{0}$ as a function of normalized pressure $p / E^{*}$ from small to full contact.

The fitted $\tilde{G}(p, \zeta)$ can now be compared with the theory. Thus, the theory of Persson predicts $\tilde{G}=G$ where,

$$
G=\frac{\pi}{4}\left(\frac{E}{1-\nu^{2}}\right)^{2} \int_{q_{L}}^{\zeta q_{0}} d q q^{3} C(q) .
$$

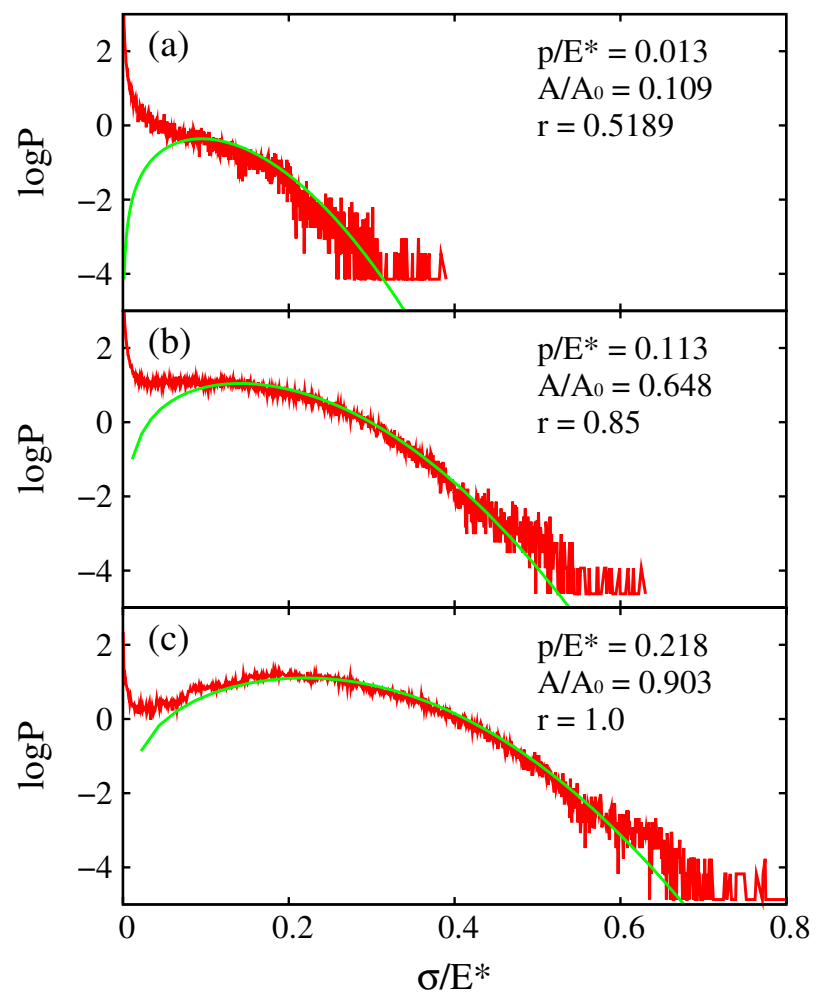

FIG. 1 (color online). The pressure distribution for $\zeta=4$ for three different nominal pressure, (a) $p / E^{*} \approx 0.013$, (b) $p / E^{*} \approx 0.113$, (c) $p / E^{*} \approx 0.218$. The pressure probability distribution becomes broader with increasing the squeezing pressure. Properly choosing the correction factor (see Fig. 2) makes the numerical results in good agreement with Persson's contact mechanics theory.

Figure 2(a) shows the ratio $r=\tilde{G} / G$ which is referred to as correction factor. Note that $r$ increases from $\approx 0.51$ to 1 as the squeezing pressure $p$ increases from zero to infinite (i.e., the normalized contact area $A / A_{0}$ increases from zero to 1$)$. Since the contact area for small load is proportional to $\sim 1 / \sqrt{G}$, it follows that the theory for small load predicts a contact area about $\sim 30 \%$ smaller than that deduced from the MD simulation. This is slightly larger than what has been found in earlier numerical simulations. Thus, the finite element calculations of Hyun and Robbins [22] and the Green's function molecular dynamics study of Campana and Müser [13] gives $r \approx 0.64$, corresponding to a contact area about $\sim 20 \%$ larger than that predicted by the Persson theory. Similarly, the study of Hönig [23] gives $r \approx 0.56$ for small load. However, none of the computer simulations can be considered as perfectly converged, so the difference between theory and fully converged numerical simulation may be smaller than that indicated by the numbers given above.

Recently Persson theoretically derived the relation between the average interfacial separation $\bar{u}$ and the applied normal squeezing pressure $p$ [18]. For nonadhesive interaction and small applied pressure, $p \sim e^{-\bar{u} / u_{0}}$, in a good 


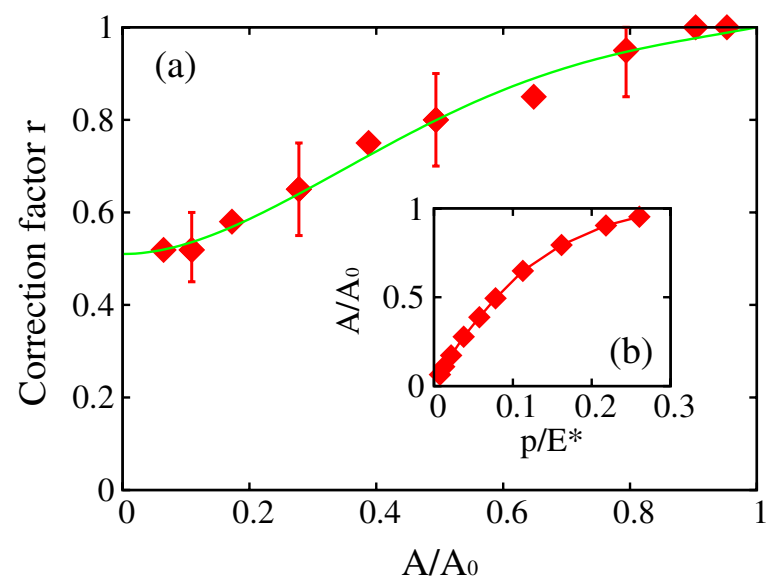

FIG. 2 (color online). (a) Correction factor $r$ as a function of the contact area ratio $A / A_{0}$. The points are simulation results, which have been fitted by the function $f(x)=a+b x^{2}+c x^{3}+$ $d x^{4}$ under the condition $d=1-a-b-c$ (solid line). The corresponding coefficients $a, b, c$ are $0.51,2.5,-3.3$, respectively. (b) The contact area ratio $A / A_{0}$ as a function of squeezing pressure normalized by effective elastic modulus.

agreement with recent experimental observations [24,25]. Here we numerically calculate the average interfacial separation with different squeezing pressure with molecular dynamics. In Fig. 3 we show the natural logarithm of the normalized average pressure $p / E^{*}$, as a function of the normalized interfacial separation $\bar{u} / h_{\text {rms }}$. We show results for the magnification $\zeta=4$ (open circles) and $\zeta=216$ (solid squares). Since the atoms interact with a long-range repulsive $\sim r^{-12}$ pair potential, it is possible to squeeze the surfaces closer to each other than what corresponds to $\bar{u}=$ 0 . This explains why simulation data points occur also for $\bar{u}<0$.

In Fig. 4 we compare the MD results from Fig. 3 (open circles) with the theory presented in Ref. [18] using the same surface roughness power spectrum (and other parameters) as in the MD calculation. The theory is in good

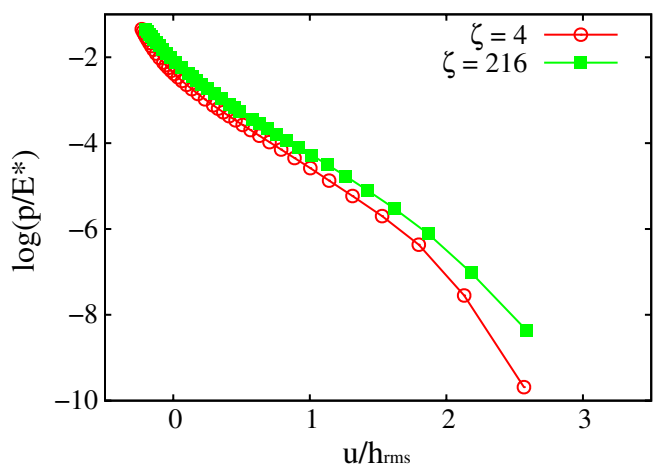

FIG. 3 (color online). An elastic block squeezed against a rigid rough substrate. The natural logarithm of the normalized average pressure $\log \left(p / E^{*}\right)$, as a function of normalized interfacial separation $\bar{u} / h_{\text {rms }}$ on different magnifications $\zeta=4$ and $\zeta=$ 216. agreement with the numerical data for $0.2<\bar{u} / h_{\text {rms }}<2$. For $\bar{u} / h_{\text {rms }}<0.2$ the two curves differ because of the reason discussed above; i.e., the "soft" potential used in the MD simulation allows the block and substrate atoms to approach each other beyond $u(\mathbf{x})=0$, while in the analytical theory the potential is infinite for $u(\mathbf{x})<0$ and zero for $u(\mathbf{x})>0$. The difference between the theory and the MD results for $\bar{u} / h_{\mathrm{rms}}>2$ is due to a finite size effect. That is, since the MD calculations use a very small system, the highest asperities are only $\sim 3 h_{\mathrm{rms}}$ above the average plane (see the height distribution in Ref. [26]), and for large $\bar{u}$ very few contact spots will occur, and, in particular, for $\bar{u}>3 h_{\text {rms }}$ no contact occurs and $p$ must vanish. In the analytical theory, the system size is assumed to be infinite. Even for a Gaussian distribution of asperity height, there will always be infinitely many infinitely high asperities. Contact will occur at arbitrarily large separation $\bar{u}$, and the asymptotic relation $\bar{u} \sim \log p$ will hold for arbitrarily large $\bar{u}$ at small squeezing pressures $p$.

Let us now discuss the probability distribution of interfacial separation, defined by

$$
P_{u}=\langle\delta(u-u(\mathbf{x}))\rangle,
$$

where $\langle\ldots\rangle$ is ensemble average. This function is shown in Fig. 5 for three different loads and two different magnifications. Note that the distributions of interfacial separations observed at low and high magnifications are similar for $u>5 \AA$. This result is expected since mainly the longwavelength, large amplitude roughness will determine the separation between the surfaces when the separation is large. The quantity $P_{u}$ has many important applications. For example, for lubricated contact at low sliding velocity, one may estimate the contribution from shearing the liquid film to the (nominal) frictional stress using

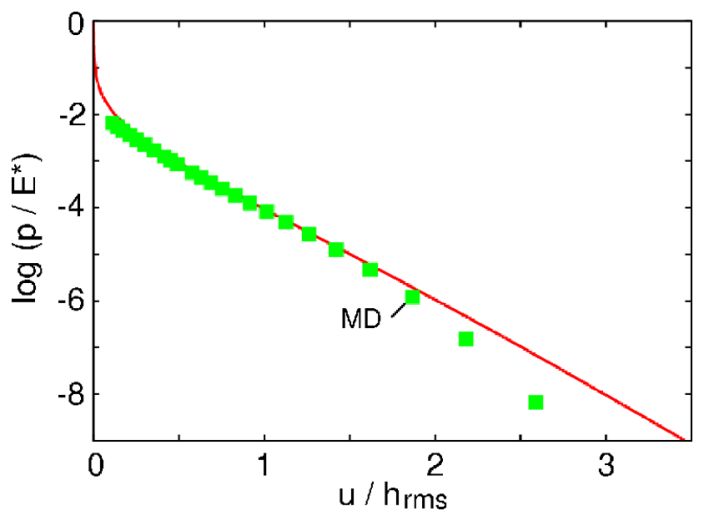

FIG. 4 (color online). The relation between the natural logarithm of the squeezing pressure $p$ (normalized by $E^{*}$ ) and the interfacial separation $\bar{u}$ (normalized by the root-mean-square roughness amplitude $h_{\text {rms }}$ ) for an elastic solid squeezed against a rigid surface. The theory curve (solid line) has been calculated using the theory presented in Ref. [18] with $\gamma=0.42$. 


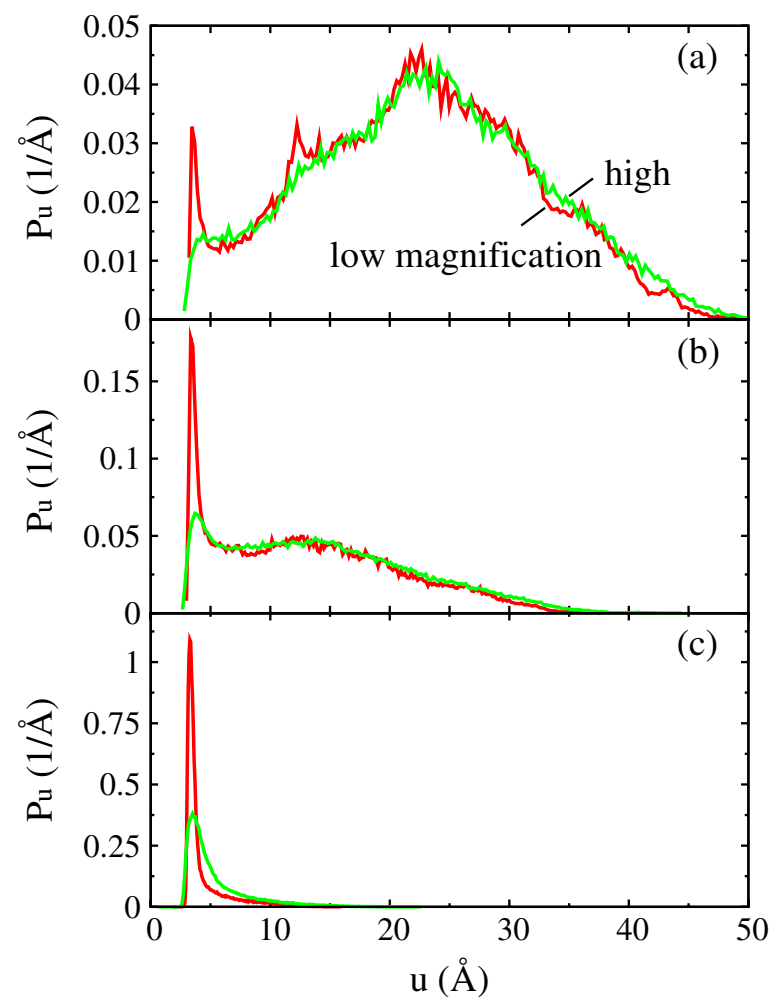

FIG. 5 (color online). Probability distribution of interfacial separation under different pressure (a) $p / E^{*} \approx 0.002$, (b) $p / E^{*} \approx 0.013$, (c) $p / E^{*} \approx 0.113$, at low and high magnifications, respectively. For low pressure, the interfacial separation probability distribution on low and high magnification is similar for $u>5 \AA$. This result is expected since mainly the longwavelength, large amplitude roughness will determine the separation between the surfaces when the separation is large.

$$
\tau \approx \eta v \int_{u_{c}}^{\infty} d u \frac{P_{u}}{u},
$$

where $\eta$ is the viscosity and $v$ the sliding velocity, and where $u_{c}$ is a cutoff separation of order nanometer (continuum fluid dynamics is not valid for liquid films thinner than a few nanometers). Another important application is for estimating the fluid leaking through sealing [26].

To summarize, we have performed a molecular dynamics (MD) study of the contact between an elastic block with a flat surface and a rigid substrate with a randomly rough surface. The interfacial pressure distribution agrees well with the analytical theory of Persson. We have also calculated the area of real contact and the interfacial separation as a function of load from small to full contact, and compared the results with the analytical theory. For not too large or too small squeezing pressures, the MD results show that the interfacial separation $\bar{u}$ depends logarithmically on the squeezing pressure $\bar{u} \sim \log p$, in a good agree- ment with the analytical theory and with experimental observation. Finally, we have studied the probability distribution of interfacial separation for different pressure and different magnifications. The present results may be of great importance for soft solids, e.g., rubberlike material, or very smooth surfaces.

We thank Ugo Tartaglino for many useful discussions related to the MD-simulation.

[1] F. P. Bowden and D. Tabor, The Friction and Lubrication of Solids (Oxford press, New York, 1986).

[2] B. N. J. Persson, Sliding Friction: Physical Principles and Applications (Springer, Heidelberg, 2000), 2nd ed.

[3] M. Borri-Brunetto, B. Chiaia, and M. Ciavarella, Comput. Methods Appl. Mech. Eng. 190, 6053 (2001).

[4] S. Hyun, L. Pei, J. F. Molinari, and R. O. Robbins, Phys. Rev. E 70, 026117 (2004).

[5] J.N. Israelachvili, Intermolecular and Surface Forces (Academic Press, London, 1992), 2nd ed.

[6] C. Yang, U. Tartaglino, and B. N. J. Persson, J. Phys. Condens. Matter 18, 11521 (2006).

[7] A. I. Volokitin and B. N. J. Persson, Rev. Mod. Phys. 79, 1291 (2007).

[8] N. Patir and H. S. Cheng, J. Lubr. Technol. 100, No. 1, 12 (1978); 101, No. 2, 220 (1979).

[9] A. W. Bush, R. D. Gibson, and T. R. Thomas, Wear 35, 87 (1975).

[10] J. A. Greenwood and J. B. P. Williamson, Proc. R. Soc. A 295, 300 (1966).

[11] S. Hyun, L. Pei, J. F. Molinari, and M. O. Robbins, Phys. Rev. E 70, 026117 (2004).

[12] C. Yang, U. Tartaglino, and B. N.J. Persson, Eur. Phys. J. E 19, 47 (2006).

[13] C. Campana and M. H. Müser, Europhys. Lett. 77, 38005 (2007).

[14] J. F. Archard, Proc. R. Soc. A 243, 190 (1957).

[15] B. N. J. Persson, F. Bucher, and B. Chiaia, Phys. Rev. B 65, 184106 (2002).

[16] B. N. J. Persson, J. Chem. Phys. 115, 3840 (2001).

[17] B. N. J. Persson, Eur. Phys. J. E 8, No. 4, 385 (2002).

[18] B. N. J. Persson, Phys. Rev. Lett. 99, 125502 (2007).

[19] K. L. Johnson, Contact Mechanics (Cambridge University Press, Cambridge, England, 1985).

[20] B. N. J. Persson, O. Albohr, U. Tartaglino, A. I. Volokitin, and E. Tosatti, J. Phys. Condens. Matter 17, R1 (2005).

[21] B. N. J. Persson, Surf. Sci. Rep. 61, 201 (2006).

[22] S. Hyun and M. O. Robbins (to be published).

[23] A. Hönig, report, Jülich, 2007 (unpublished).

[24] M. Benz, K. J. Rosenberg, E. J. Kramer, and J. N. Israelachvili, J. Phys. Chem. B 110, 11884 (2006).

[25] L. Pei, S. Hyun, J.F. Molinari, and M. O. Robbins, J. Mech. Phys. Solids 53, 2385 (2005).

[26] B. N. J. Persson and C. Yang (to be published). 